\title{
Efeito agudo da crioterapia e diatermia na flexibilidade e força muscular de isquiotibiais
}

\author{
Acute effect of cryotherapy and diathermy in the hamstring flexibility and muscle strength
}

\author{
Marcelo Baptista Döhnerta, Mariana dos Santos Oliveirab ${ }^{\text {, Rita Fagundes Hoffmann }}{ }^{c}$ \\ a Fisioterapeuta. Doutor em Ciências da Saúde pela UNESC-SC. Professor do Curso de Fisioterapia da Universidade Luterana do Brasil/Torres/RS/Brasil. \\ b Fisioterapeuta. Pós graduanda em Atenção Geriátrica Integrada, Pontifícia Universidade Católica do Rio Grande do Sul (PUCRS), Brasil. \\ c Fisioterapeuta graduada pela Universidade Luterana do Brasil/Torres/RS/Brasil. Terra de Areia, Rio Grande do Sul, Brasil.
}

\begin{tabular}{l|l} 
RESUMO Objetivo: Verificar o efeito agudo da crioterapia e da diatermia por ondas curtas na flexibilidade e força muscular
\end{tabular} de isquiotibiais de adultas jovens.

Materiais e Métodos: Quarenta e cinco voluntárias foram randomizadas em grupo controle, grupo crioterapia e grupo diatermia por ondas curtas. Os sujeitos foram submetidos a uma única aplicação de crioterapia ou diatermia por ondas curtas. Foram avaliadas, pré e pós intervenção, a flexibilidade através do banco de Wells e do ângulo de extensão ativa do joelho, e a força muscular através da dinamometria. As variáveis foram analisadas por meio do teste $t$ de Student e ANOVA.

Resultados: Verificou-se um aumento significativo da flexibilidade dos isquiotibiais, tanto através do banco de Wells $(24,50 \pm 6,88 \mathrm{~cm}$ para $26,87 \pm 8,02 \mathrm{~cm})(p=0,002)$, quanto na extensão ativa do joelho $(162,60 \pm 7,76$ graus para $165,53 \pm 6,38$ graus) ( $p=0,015)$ no grupo diatermia. O grupo crioterapia mostrou melhora da flexibilidade no banco de Wells $(23,27 \pm 6,33 \mathrm{~cm}$ para $25,53 \pm 6,13 \mathrm{~cm})(p=0,002)$. Não ocorreu aumento significativo da força muscular em nenhum dos grupos de intervenção. Não foi verificada diferença entre os grupos do estudo.

Conclusão: Tanto a diatermia quanto a crioterapia melhoraram, de forma aguda, a flexibilidade dos isquiotibiais em mulheres adultas jovens e saudáveis. A termoterapia através da diatermia por ondas curtas mostrou-se mais efetiva nessa melhora. Ambos os recursos não alteraram a força muscular produzida pelos isquiotibiais.

Palavras-chave: crioterapia; diatermia; flexibilidade; força muscular.

Objective: To investigate the acute effect of cryotherapy and short-wave diathermy on flexibility and muscle strength in the hamstrings of young women.

Materials and Methods: Forty-five volunteers were randomized into a control group, a cryotherapy group and a short-wave diathermy group. The subjects underwent a single session of cryotherapy or short-wave diathermy. Flexibility through the Wells bench and the active knee-extension end range and muscle strength through dynamometry were assessed pre and post treatment. The variables were analyzed through the Student $t$ test and ANOVA.

Results: A significant increase in the hamstring flexibility was found using both the Wells bench $(24.50 \pm 6.88 \mathrm{~cm}$ to $26.87 \pm 8.02 \mathrm{~cm})$ $(p=0.002)$ and the active knee-extension end range $(162.60 \pm 7.76$ degrees to $165.53 \pm 6.38$ degrees $)(p=0.015)$ in the diathermy group. The cryotherapy group showed improvement in flexibility with the Wells bench test $(23.27 \pm 6.33 \mathrm{~cm}$ to $25.53 \pm 6.13 \mathrm{~cm})$ $(p=0.002)$. No change was observed in the hamstring muscle strength in either treatment group. The control group showed no difference in any of the variables. No differences were observed between groups.

Conclusion: Both short-wave diathermy and cryotherapy acutely improved hamstring flexibility in healthy young women. Thermotherapy by shortwave diathermy was more effective in this case. Both procedures had no effect on the force produced by the hamstring muscle.

Keywords: cryotherapy; diathermy; flexibility; muscle strength. 


\section{INTRODUÇÃO}

Flexibilidade e força muscular são variáveis, normalmente, modificadas em pacientes e sujeitos que apresentam algum tipo de disfunção musculoesquelética. A flexibilidade muscular é definida como a habilidade de um músculo alongar-se, permitindo que a articulação se movimente através de toda a amplitude de movimento (ADM), irrestrita e sem dor. A perda da flexibilidade e consequente redução da ADM ocorre, entre diversas condições, pela diminuição da capacidade de um músculo deformar-se, relacionada à perda de força muscular e à presença de encurtamentos ${ }^{1-5}$. A redução da flexibilidade, principalmente em isquiotibiais, pode gerar desequilíbrio postural, déficit da ADM do joelho, do quadril e da coluna lombar e dificuldade em realizar movimentos amplos ${ }^{3,5}$. Essas alterações contribuem para lesões e disfunções musculoesqueléticas ${ }^{2,6}$.

Diversas condições articulares e periarticulares levam à perda ou à redução da força muscular. A força muscular é conceituada como a capacidade do músculo esquelético produzir tensão e torque máximo. Várias técnicas são usadas para maximizar o treinamento de força muscular ${ }^{7,8}$. Entre elas está o aquecimento, que visa aumentar a temperatura muscular, aumentar o metabolismo energético, dar elasticidade ao tecido conjuntivo, aumentar o débito cardíaco e a velocidade de transmissão do impulso nervoso, diminuir a viscosidade do sistema miotendíneo, redistribuir o fluxo sanguíneo e melhorar a difusão do oxigênio disponível nos músculos ${ }^{7,8}$. Alguns fatores determinam a força muscular em cada indivíduo, tais como: gênero, estado hormonal, neurológicos, nutricionais, aspectos ambientais e grau de atividade física ${ }^{7,8}$.

Alguns recursos físicos e eletrofísicos são utilizados no tratamento das disfunções musculoesqueléticas. Entre estes, estão a diatermia por ondas curtas e a crioterapia. A geração de calor é o principal efeito da diatermia por ondas curtas. O seu uso prévio em programas de alongamentos é sugerido por proporcionar melhores resultados na flexibilidade e diminuir o desconforto durante as sessões de alongamento ${ }^{1,9,10}$. A implementação clínica do calor é sugerida por aumentar a extensibilidade das fibras colágenas, favorecendo o relaxamento das propriedades mecânicas do músculo ${ }^{1,9,10}$.

Crioterapia é o termo que se refere à aplicação de frio sobre o organismo, com fins terapêuticos, geralmente, empregada no tratamento da dor em afecções musculoesqueléticas agudas, processos inflamatórios e contraturas musculares ${ }^{11,12}$. O resfriamento local ocorre por transferência de calor corporal (condução, convecção e evaporação) para um elemento externo cuja temperatura é mais baixa ${ }^{11,12}$. Muitos estudos avaliaram a influência da crioterapia na flexibilidade ${ }^{11,12}$. Esta pode reduzir a velocidade de condução nervosa, diminuir a descarga do fuso muscular, diminuindo-se, por conseguinte, a interferência desse estímulo na tensão muscular, permitindo maior flexibilização ao músculo ${ }^{1,10,13,14}$.

O nosso estudo teve como objetivo verificar os efeitos agudos do resfriamento e do calor profundo sobre as propriedades de extensibilidade e força muscular em isquiotibiais de indivíduos jovens e saudáveis do sexo feminino.

\section{MATERIAIS E MÉTODOS}

\section{Delineamento}

Este foi um estudo controlado randomizado cego.

\section{Seleção da amostra}

A amostra foi composta por 45 estudantes do sexo feminino do curso de fisioterapia da ULBRA-Torres. O estudo foi realizado na clínica escola de fisioterapia, no período de abril e maio de 2012. A escolha por sujeitos do sexo feminino baseou-se na busca de uma homogeneidade da amostra utilizada quanto às variáveis do estudo. Após a análise dos critérios de inclusão e exclusão, 45 estudantes preencheram os requisitos para ingressarem no estudo.

\section{Randomização}

Após a avaliação inicial e preenchidos os critérios de elegibilidade, os 45 sujeitos elegíveis foram divididos, através da listagem de números aleatórios fornecidos pelo pacote estatístico EPI-INFO ${ }^{\circledR}$, em três grupos: grupo I, grupo controle, com $n=15$, os quais apenas foram avaliados; grupo II, grupo crioterapia, com $n=15$, os quais foram avaliados e receberam uma aplicação da crioterapia por 20 minutos, sendo reavaliados imediatamente após; grupo III, grupo diatermia por ondas curtas, $\mathrm{n}=15$, os quais foram avaliados e receberam uma única aplicação da diatermia por ondas curtas de 20 minutos, sendo reavaliados imediatamente após.

\section{Critérios de elegibilidade}

Critérios de Inclusão: estudantes do sexo feminino com idade entre 18 e 25 anos e que consentiram em participar do estudo e assinaram o Termo de Consentimento Livre e Esclarecido. Critérios de Exclusão: história de doença sistêmica, infecciosa ou inflamatória reumática, história de lesão musculoesquelética e/ou cirurgia em membros inferiores, sujeitos com hipersensibilidade ao frio ou ao calor, marcapasso implantado, presença de metal nos tecidos e implante metálico no corpo, áreas hemorrágicas e/ou isquêmicas, útero grávido, tumores, infecção, tuberculose ativa, trombose venosa profunda, ferimentos abertos na 
área de aplicação, pele anestesiada, insuficiência circulatória periférica, alergia ao frio, enfermidade de Raynaud.

\section{Protocolo de avaliação}

A randomização foi realizada por pesquisadora exclusiva responsável pela aplicação da técnica terapêutica e que não realizou as avaliações e reavaliações. Estas foram efetuadas por outra pesquisadora cega, ou seja, que não sabia a que grupo pertencia o sujeito. A avaliação inicial foi realizada previamente à randomização e, nesta, foi realizada uma anamnese completa e testes de sensibilidade superficial a fim de avaliar os critérios de inclusão/exclusão.

Inicialmente, o peso corporal foi aferido utilizando-se uma balança de precisão digital, marca Filizola ${ }^{\circledR}$, previamente calibrada, estando o indivíduo com roupas leves e descalço. A medição de estatura foi efetuada na mesma balança através do estadiômetro, com o sujeito sem calçados, posição anatômica, braços estendidos ao longo do corpo e em apnéia respiratória. Após, foi calculado o índice de massa corporal, através da medição da altura e do peso corporal.

A avaliação da flexibilidade foi mensurada por dois testes específicos. Através do banco de Wells, o sujeito foi posicionado sentado, com os pés apoiados no aparelho e com as pernas estendidas ${ }^{15}$. Foi solicitado ao mesmo realizar uma flexão do tronco e do quadril com as mãos sobrepostas e apoiadas sobre a fita métrica, instalada na parte superior do banco de Wells, mantendo os joelhos em extensão durante todo o movimento ${ }^{15}$. A leitura da flexibilidade foi feita pelo avaliador quando o sujeito atingiu o ponto máximo de flexão do tronco e quadril à frente ${ }^{15}$. As medições foram realizadas três vezes consecutivas e, posteriormente, foi utilizada a mediana destas ${ }^{15}$.

Outro teste de flexibilidade utilizado foi através do ângulo de extensão ativa do joelho¹. O sujeito foi colocado em uma maca em decúbito dorsal com o quadril direito fletido a $90^{\circ}$ sustentado pelo pesquisador e o membro inferior esquerdo mantido em extensão na maca. Este posicionamento de quadril foi mantido pelo pesquisador, enquanto foi solicitado à voluntária estender, ativamente, o joelho direito ${ }^{1}$. Foi realizada, então, a goniometria final da extensão de joelho direito ao atingir a tensão sobre os isquiotibiais ${ }^{1}$. Para isso, utilizou-se um goniômetro da marca $\mathrm{CARCl}^{\circledR}$ (São Paulo, Brasil), sendo este posicionado com o eixo fixo colocado na linha média do côndilo lateral do fêmur, o braço fixo direcionado para o trocânter maior do fêmur alinhado com a coxa e o braço móvel em paralelo à perna .

A força muscular dos isquiotibiais foi mensurada por meio da dinamometria, realizada no membro inferior direito. Utilizou-se um dinamômetro portátil, do tipo push-pull, marca Chataanooga ${ }^{\circledR}$. A máxima força isométrica voluntária foi mensurada num ângulo de 60 graus de flexão de joelho, com o sujeito em decúbito ventral. A mediana das três medições realizadas foi utilizada como a máxima contração isométrica voluntária (MCIV) ${ }^{16}$.

Os testes acima descritos foram realizados imediatamente antes e após a intervenção proposta.

\section{Protocolo de Intervenção}

Ambos os protocolos de intervenção foram realizados imediatamente após a avaliação inicial. A aplicação da crioterapia foi realizada com o sujeito em decúbito dorsal, com uma bolsa de gelo acoplada no terço médio da parte posterior da coxa (músculos isquiotibiais) do membro inferior direito, por 20 minutos. Foi utilizado pacote de gelo moído com compressão local feita com uma atadura elástica.

A aplicação da diatermia por ondas curtas contínuo foi realizada por 20 minutos, com o sujeito em decúbito dorsal, de forma coplanar na parte posterior da coxa (músculos isquiotibiais) do membro inferior direito, estando os eletrodos fixados por tiras elásticas. O nível de calor utilizado foi o tolerável pelo sujeito. Utilizou-se um equipamento marca IBRAMED ${ }^{\circledR}$, modelo Thermopulse - Automatic Tuning, da clínica escola de fisioterapia da Universidade Luterana do Brasil, campus de Torres-RS, previamente calibrado.

\section{Aspectos Éticos}

O estudo foi aprovado pelo Comitê de Ética da Universidade Luterana do Brasil sob o número 2011-442H. Todas as voluntárias que participaram do estudo receberam, inicialmente, informações sobre a pesquisa e assinaram o Termo de Consentimento Livre e Esclarecido (TCLE) redigido segundo as Diretrizes e Normas Regulamentadoras de Pesquisa Envolvendo Seres Humanos, constantes da Resolução do Conselho Nacional da Saúde no 466/2012. O TCLE foi elaborado em duas vias, sendo uma para os pesquisadores e outra para a voluntária envolvida.

\section{Análise Estatística}

Inicialmente, os dados foram expressos de forma descritiva em frequência, média e desvio padrão. A normalidade de distribuição foi testada por meio do teste de Shapiro-Wilk. O teste de ANOVA seguido do post hoc de Tukey foi utilizado para testar as diferenças entre parâmetros antropométricos no início do estudo e para avaliação das variáveis de distribuição paramétrica entre os grupos de intervenção e controle. Para avaliação pré e pós-intervenção dentro de cada grupo, foi utilizado o teste $t$ de Student pareado. O nível de significância estabelecido para o teste estatístico é de $p<0,05$. Foi utilizado o SPSS (Statistical Package for the Social Sciences) versão 17.0 como pacote estatístico. 


\section{RESULTADOS}

Entre abril e maio de 2012, 45 estudantes do sexo feminino do curso de fisioterapia da ULBRA-Torres foram incluídas neste estudo. A média de idade total foi de $21,07 \pm 1,91$ anos, $161,17 \pm 6,63 \mathrm{~cm}$ de estatura, peso de $61,22 \pm 10,99 \mathrm{~kg}$ e IMC médio de $24,49 \pm 3,33$. Os grupos ficaram homogêneos entre si quanto às variáveis idade, estatura, peso e IMC (Tabela 1).

Observou-se, por meio do teste $t$ de Student que, tanto no grupo crioterapia quanto no grupo diatermia, houve aumento significativo da flexibilidade para teste com o banco de Wells do pré para o pós-intervenção. O grupo crioterapia passou de $23,27 \pm 6,33 \mathrm{~cm}$ para $25,53 \pm 6,13 \mathrm{~cm}(p=0,002)$. Já o grupo diatermia aumentou de $24,50 \pm 6,88 \mathrm{~cm}$ para $26,87 \pm 8,02 \mathrm{~cm}(p=0,002)$ (Tabela 2).

$\mathrm{Na}$ avaliação da flexibilidade pelo teste de extensão ativa do joelho, apenas o grupo diatermia mostrou uma melhora significativa no teste $t$ de Student, passando de 162,60土 7,76 graus para $165,53 \pm 6,38$ graus $(p=0,015)$. Em relação à força muscular, não foram verificadas alterações significativas da fase pré para pós-intervenção em todos os grupos de estudo (Tabela 2).
Analisando as diferenças entre os grupos de estudo tanto no pré quanto pós-intervenção por meio da análise de variância (ANOVA) one way, não se constataram diferenças estatísticas entre os grupos quanto à força, flexibilidade e mobilidade articular de joelho.

\section{DISCUSSÃO}

No presente estudo, verificaram-se os efeitos agudos locais do resfriamento e do calor profundo sobre as propriedades de flexibilidade e força muscular em isquiotibiais. Observou-se que, tanto a crioterapia quanto a diatermia, aumentaram, significativamente, a flexibilidade de isquiotibiais do pré para o pós-intervenção avaliada por meio do banco de Wells. Porém, na avaliação da mesma, com extensão ativa de joelho e quadril fletido a $90^{\circ}$, apenas o grupo diatermia mostrou melhora significativa. Os resultados obtidos assemelham-se aos de Brasileiro et al. ${ }^{1}$, em que 40 voluntários foram divididos, aleatoriamente, em quatro grupos (grupo controle, grupo alongamento, grupo alongamento precedido da aplicação de crioterapia e grupo alongamento precedido de aquecimento com diatermia por ondas curtas). Os autores analisaram a influência do resfriamento e do aquecimento muscular local sobre

Tabela 1. Caracterização da amostra do estudo.

\begin{tabular}{lccccc}
\hline \multicolumn{1}{c}{ Variável } & $\begin{array}{c}\text { Total } \\
(\mathbf{n}=\mathbf{4 5})\end{array}$ & $\begin{array}{c}\text { Grupo Controle } \\
(\mathbf{n}=\mathbf{1 5})\end{array}$ & $\begin{array}{c}\text { Grupo Crioterapia } \\
(\mathbf{n = 1 5 )}\end{array}$ & $\begin{array}{c}\text { Grupo Diatermia } \\
(\mathbf{n}=\mathbf{1 5})\end{array}$ & \multicolumn{1}{c}{ Valor de $\boldsymbol{p}^{*}$} \\
\hline Idade (anos) & $21,07 \pm 1,91$ & $21,0 \pm 2,0$ & $20,87 \pm 1,4$ & $21,33 \pm 2,32$ & 0,796 \\
Peso (Kg) & $61,22 \pm 10,99$ & $58,2 \pm 9,26$ & $63,3 \pm 10,98$ & $62,13 \pm 12,55$ & 0,418 \\
Estatura $(\mathrm{cm})$ & $161,17 \pm 6,63$ & $160,73 \pm 5,92$ & $161,33 \pm 6,9$ & $161,03 \pm 7,42$ & 0,918 \\
IMC $\left(\mathrm{Kg} / \mathrm{m}^{2}\right)$ & $24,49 \pm 3,33$ & $22,33 \pm 3,08$ & $24,33 \pm 3,49$ & $23,8 \pm 3,27$ & 0,238 \\
\hline
\end{tabular}

Valores expressos em média e desvio padrão.

* Teste ANOVA.

Tabela 2. Análise de flexibilidade e força muscular de isquiotibiais direito pré e pós intervenção.

\begin{tabular}{lccc}
\hline & $\begin{array}{c}\text { Flexibilidade } \\
(\mathbf{c m})\end{array}$ & $\begin{array}{c}\text { Extensão ativa de joelho } \\
\text { (graus) }\end{array}$ & $\begin{array}{c}\text { Força muscular } \\
\mathbf{( k g )}\end{array}$ \\
\hline $\begin{array}{l}\text { Grupo Controle }(\mathrm{n}=15) \\
\text { Pré }\end{array}$ & $25,63 \pm 7,24$ & $164,93 \pm 7,16$ & $18,07 \pm 4,18$ \\
$\quad$ Pós & $25,70 \pm 7,14$ & $164,73 \pm 6,99$ & $18,13 \pm 4,15$ \\
$\quad$ Valor de $p^{*}$ & 0,334 & 0,334 & 0,334 \\
Grupo Crioterapia $(\mathrm{n}=15)$ & & & \\
Pré & $23,27 \pm 6,33$ & $162,67 \pm 7,88$ & $20,40 \pm 3,18$ \\
Pós & $25,53 \pm 6,13$ & $164,40 \pm 6,20$ & $20,40 \pm 3,30$ \\
Valor de $p^{*}$ & 0,002 & 0,259 & 1,000 \\
Grupo Diatermia $(\mathrm{n}=15)$ & & & $21,00 \pm 3,59$ \\
Pré & $24,50 \pm 6,88$ & $162,60 \pm 7,76$ & $20,60 \pm 3,70$ \\
Pós & $26,87 \pm 8,02$ & $165,53 \pm 6,38$ & 0,688 \\
\hline Valor de $p^{*}$ & 0,002 & 0,015 & \\
\hline
\end{tabular}

* Teste $t$ de Student pareado. $p \leq 0,05$. 
a extensibilidade dos músculos isquiotibiais. Com base nisso, verificaram que os grupos de intervenção prévia com calor e frio aumentaram a extensibilidade muscular tanto no efeito agudo quanto no crônico. Porém, no efeito agudo, foi observado maior ganho de extensibilidade no grupo crioterapia quando comparado aos grupos somente alongamento ou que associou alongamento e aquecimento prévio. Contrariando os achados deste estudo, Signori et al. ${ }^{17}$ analisaram o efeito de agentes térmicos aplicados de forma preparatória a um programa de alongamentos na flexibilidade dos músculos isquiotibiais encurtados em 42 voluntários saudáveis divididos em quatro grupos (grupo controle, grupo hipotermia mais alongamentos, grupo hipertermia mais alongamentos e grupo alongamentos). O estudo mostrou que todos os grupos que realizaram alongamento apresentaram melhora significativa da flexibilidade e não houve diferenças entre os grupos. Os autores concluíram que o aumento da flexibilidade deveu-se aos alongamentos, sendo independente da aplicação prévia de hipertermia e/ou hipotermia por condução. Cabe salientar que nosso estudo avaliou apenas os efeitos agudos de calor e frio sobre as propriedades dos músculos isquiotibiais sem a associação de nenhum tipo de conduta terapêutica cinesioterapêutica. Os efeitos da alteração da temperatura sobre os diversos tipos de tecido conjuntivo modificam a sua propriedade. Knight et al. ${ }^{18}$ afirmaram que "a rigidez do tecido conjuntivo aumenta e a sua extensibilidade diminui conforme a temperatura declina", sugerindo que "o resfriamento e o alongamento associados são prejudiciais quando se deseja aumentar a extensibilidade do tecido conjuntivo". Entretanto, o aumento da flexibilidade pelo resfriamento se dá devido à diminuição da descarga fusal, à diminuição do espasmo muscular, à redução do reflexo de estiramento e à redução do limiar de excitabilidade dolorosa. A diminuição da descarga fusal e a redução do reflexo de estiramento acontecem por meio da redução do input sensorial do fuso muscular, que é responsável pela facilitação do estímulo motor e pelo aumento da tensão do músculo agonista. Uma vez reduzida a descarga fusal, reduz-se, por conseguinte, a interferência deste estímulo na tensão muscular ${ }^{1,13,17}$. Outro fator relevante, favorável ao resfriamento, seria a diminuição do limiar de sensibilidade dolorosa ao alongamento por meio da redução da velocidade de condução nervosa. Reduzida a limitação álgica, ocorre um aumento da tolerância às manobras de alongamento, permitindo uma flexibilização mais efetiva. Frequentemente, observa-se que a limitação álgica, durante as manobras de alongamento, precede a limitação tecidual (mecânica). Assim, a sensação subjetiva de desconforto, na região posterior da coxa, pode reduzir a eficiência da manobra. Os efeitos da crioterapia sobre a velocidade de condução nervosa prevaleceriam sobre as alterações na extensibilidade dos tecidos ${ }^{1,10}$.

A diatermia por ondas curtas intervém na flexibilidade muscular pelo fato de ser um recurso que proporciona aquecimento de tecidos profundos, aumento de temperatura muscular local em torno de $4^{\circ}$ e $4,6^{\circ}$ em uma profundidade de três a cinco centímetros e que permanece em torno de cinco minutos. Este aumento da temperatura local proporciona diminuição do limiar de excitabilidade nervosa, aumento do fluxo sanguíneo, aumento do metabolismo tecidual, diminuição da atividade intrafuso muscular e promove o relaxamento muscular e o aumento da extensibilidade das fibras colágenas, alterando as propriedades viscoelásticas e mecânicas do tecido muscular, proporcionando benefícios à flexibilidade de músculos encurtados ${ }^{1,10,16,17,18}$.

Não são claros, até o momento, os efeitos terapêuticos do calor profundo e da crioterapia sobre a força muscular. No presente estudo, avaliou-se a força muscular dos isquiotibiais, por meio da dinamometria manual, considerando a MCIV dos isquiotibiais. Não foram verificadas modificações, estatisticamente significativas, na força muscular entre os grupos de estudo após as intervenções. Silva et al. ${ }^{20}$ avaliaram a influência do frio no pico de torque da musculatura flexora do joelho. O efeito térmico de resfriamento diminuiu o desempenho muscular. Concluíram que vários foram os mecanismos, dentre eles, a diminuição da velocidade de condução nervosa e/ou muscular, a redução da excitabilidade do motoneurônio central, a diminuição da sensibilidade ao cálcio da actina-miosina ou a lentidão de todos os processos químicos ocorridos no músculo. Também se deve considerar o tipo de fibra muscular e a localização do músculo, pois, quanto mais próximas ao tronco, os músculos apresentam uma temperatura média de $4,1^{\circ} \mathrm{C}$ a mais que a dos músculos distais. Acreditou-se que o resfriamento prolongado diminuiu o pico de torque pela interferência com o recrutamento das unidades motoras e da velocidade de condução. Crystal Naomi et al. ${ }^{21}$ avaliaram o pico de torque extensor do joelho após resfriamento e não foi verificada uma alteração significativa deste após resfriamento ${ }^{21}$. Mustalampi et al. ${ }^{22}$ examinaram os efeitos agudos da crioterapia em 39 sujeitos saudáveis sobre a propriedade muscular do quadríceps. Estes autores observaram que o quadríceps tornou-se tenso, rígido e pouco elástico, após a aplicação, e que suas propriedades demoraram mais de 15 minutos para se normalizarem ${ }^{22}$. Em 2010, Akyol et al. ${ }^{23}$ estudaram a eficácia da diatermia por ondas curtas, associada ou não a exercícios isocinéticos, na dor, função, força muscular e qualidade de vida em pacientes com osteoartrite de joelho. Em relação à força muscular, verificaram que não houve diferença significativa entre o grupo que associou ondas curtas aos exercícios isocinéticos 
para fortalecimento muscular e o grupo que utilizou apenas exercícios isocinéticos para fortalecimento muscular.

Cetin et al. ${ }^{24}$ avaliaram os efeitos terapêuticos de agentes físicos administrados antes de exercícios isocinéticos na dor, incapacidade, a força muscular em mulheres com osteoartrite de joelho. Foram utilizados, antes dos exercícios, a eletroestimulação nervosa transcutânea, o ultrassom ou a diatermia por ondas curtas. Estes autores verificaram que houve aumento no desempenho do exercício, redução da dor e melhora na função em todos os grupos. Porém, na eletroestimulação nervosa transcutânea e na diatermia por ondas curtas, os resultados foram melhores, quando comparados ao ultrasom. Já Rattanachaiyanont et al. ${ }^{25}$ avaliaram o efeito da diatermia por ondas curtas associado a um programa de exercícios para osteoartrite de joelho em mulheres. Concluíram que não houve melhora na associação da diatermia por ondas curtas em um programa de exercícios para mulheres com osteoartrite de joelho. Estes estudos relatados anteriormente, apesar de terem submetidos os sujeitos a um programa de treinamento, diferentemente do nosso estudo, corroboram com os nossos achados no quesito força muscular associado ao calor profundo, em que verificamos que a diatermia por ondas curtas não altera de forma aguda a força muscular de isquiotibiais em mulheres jovens. Contrariando os estudos anteriores, Petrofsky et al. ${ }^{26}$ abordaram 20 sujeitos com calor e crioterapia no quadríceps femoral e foi constatada uma diminuição da tensão sobre as estruturas moles apenas com o uso do calor ${ }^{26}$.

Para o presente estudo, foram selecionados apenas sujeitos do sexo feminino com idades entre 18 e 25 anos. O índice de massa corporal (IMC) foi um dos itens avaliados devido à diatermia por ondas curtas e à crioterapia serem agentes físicos, que dependem do tipo e da profundidade dos tecidos para seu melhor efeito. O tecido adiposo não apresenta a mesma capacidade de condutividade apresentada pelo tecido muscular. Portanto, sujeitos com IMC muito alto podem apresentar resultados discrepantes quanto ao efeito dos agentes térmicos utilizados. Neste estudo, o IMC total

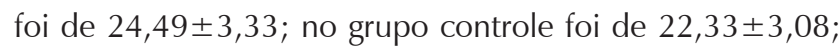
no grupo crioterapia, de 24,33 $\pm 3,49$; e no grupo diatermia, de $23,8 \pm 3,27$. Não foram verificadas diferenças entre os grupos de estudo. Segundo Carvalho et al. ${ }^{27}$, as mulheres apresentam uma melhor flexibilidade quando comparadas aos homens. Já para Petrofsky et al. ${ }^{26}$, mulheres apresentaram maior flexibilidade e elasticidade de tecidos moles do que os homens com qualquer temperatura aplicada.

As implicações deste estudo devem voltar-se para a prática clínica do fisioterapeuta. São frequentes as indicações de uso da crioterapia e, principalmente, de calor profundo como coadjuvante em manobras cinesioterapêuticas de alongamento e de reforço muscular, bem como utilizados por alguns profissionais como forma terapêutica isolada. $\mathrm{O}$ nosso estudo não permite fazer qualquer inferência aos efeitos destes recursos ao longo de um tratamento devido ao fato de ter sido analisado apenas o efeito imediato dos mesmos. Porém, deve ser observado que tais aplicações aumentam excessivamente o tempo de intervenção, ampliando também os gastos financeiros com o tratamento e a complexidade do atendimento. Como o ganho imediato de flexibilidade dos isquiotibiais, em favor do calor profundo, não se reflete, cronicamente, em ganho real de flexibilidade, quando utilizado de forma isolada, e que ambos os recursos não provocam modificação aguda da força muscular, podese entender que tais intervenções podem tornar-se, nestes objetivos terapêuticos, desnecessárias. Dessa forma, apenas a eficiência das manobras cinesioterapêuticas de reforço muscular e alongamento podem determinar possíveis alterações nas propriedades viscoelásticas dos músculos ao longo de um processo terapêutico.

Este estudo apresenta algumas limitações à capacidade de extrapolação dos resultados. O fato de utilizar apenas mulheres jovens e saudáveis não permite uma análise do comportamento fisiológico desses recursos quando utilizados em determinadas afecções musculoesqueléticas. Da mesma forma, neste estudo, apenas foi analisado o comportamento agudo da aplicação do calor profundo e da crioterapia, não permitindo a extrapolação destes resultados quando analisado um efeito tardio e acumulativo destes recursos ao longo de um protocolo terapêutico. Portanto, sugerem-se novos estudos para que se possa avaliar as características da aplicação de recursos térmicos sobre as propriedades musculares.

O presente estudo, dentro das condições experimentais propostas, sugere que os efeitos agudos da crioterapia e termoterapia, por meio da diatermia por ondas curtas sobre as propriedades de extensibilidade muscular, são favorecidos pela aplicação de ambos os recursos estudados, não havendo diferença entre os grupos quando analisada a capacidade de ativação muscular. Esses achados devem limitar-se a sujeitos com integridade no sistema neuromotor, não devendo ser extrapolados, por exemplo, para indivíduos com comprometimento neurológico. Recomenda-se que estudos semelhantes sejam realizados, sobretudo avaliando os efeitos crônicos destas técnicas e sua associação a manobras cinesioterapêuticas.

\section{REFERÊNCIAS}

1. Brasileiro JS, Faria AF, Queiroz LL. Influência do resfriamento e do aquecimento local na flexibilidade dos músculos isquiotibiais. Braz J Phys Ther. 2007;11(1):57-61. https://doi.org/10.1590/S141335552007000100010 
2. Aquino CF, Gonçalves GGP, Fonseca ST, Mancini MC. Análise da relação entre flexibilidade e rigidez passiva dos isquiotibiais. Rev Bras Med Esporte. 2006;12:(4):195-200. https://doi.org/10.1590/ S1517-86922006000400006

3. Carregaro RL, Silva LCCB, Gil Coury HJC. Comparação entre dois testes clínicos para avaliar a flexibilidade dos músculos posteriores da coxa. Braz J Phys Ther. 2007;11(2):139-45. https://doi.org/10.1590/ S1413-35552007000200009

4. Maciel ACC, Câmara SMA. Influência da estimulação elétrica nervosa transcutânea (TENS) associada ao alongamento muscular no ganho de flexibilidade. Braz J Phys Ther. 2008;12(5):373-8. https:// doi.org/10.1590/S1413-35552008000500006

5. Rizzi PRS, Leal RM, Vendrusculo AP. Efeito da hidrocinesioterapia na força muscular e na flexibilidade em idosas sedentárias. Fisioter Mov. 2010;23:(4):535-43. https://doi.org/10.1590/S010351502010000400004

6. Zenewton ASG, Medeiros CAS, Dantas AVR, Souza TO. Influência da frequência de alongamento utilizando facilitação neuromuscular proprioceptiva na flexibilidade dos músculos isquiotibiais. Rev Bras Med Esporte. 2007;13:(1):33-8. https://doi.org/10.1590/S151786922007000100008

7. Albuquerque CV, Maschio JP, Gruber CR, de Souza RM, Hernandez S. Efeito agudo de diferentes formas de aquecimento sobre a força muscular. Fisioter Mov. 2011;24(2):221-9. https://doi.org/10.1590/ S0103-51502011000200003

8. Schneider P, Rodrigues LA, Meyer F. Dinamometria computadorizada como metodologia de avaliação de força muscular de meninos e meninas em diferentes estágios de maturidade. Rev Paul Educ Fís. 2002; 16(1):35-42.

9. Lentell G, Hetherington T, Eagan J, Morgan M. The use of thermal agents to influence the effectiveness of a low-load prolonged stretch. J Orthop Sports Phys Ther. 1992;16:200-7. https://doi.org/10.2519/ jospt.1992.16.5.200

10. Silva SA, Oliveira DJ, Jaques MJN, Araújo RC. Efeito da crioterapia e termoterapia associados ao alongamento estático na flexibilidade dos músculos isquiotibiais. Motricidade. 2010;6(4):55-62. https:// doi.org/10.6063/motricidade.6(4).138

11. Schaser KD, Disch AC, Stover JF, Lauffer A, Bail HJ, Mittlmeier T. Prolonged superficial local cryotherapy attenuates microcirculatory impairment, regional inflammation, and muscle necrosis after closed soft tissue injury in rats. Am J Sports Med. 2007;35:93-102. https:// doi.org/10.1177/0363546506294569

12. Agne JE. Eletrotermoterapia: teoria e prática. Santa Maria: Orium; 2005.

13. Busarello FO, Souza FT, Paula GF. Ganho de extensibilidade dos músculos isquiotibiais comparando o alongamento estático associado ou não à crioterapia. Fisioter Mov. 2011;24(2):247-54. https://doi.org/10.1590/S0103-51502011000200006

14. Burke DG, Holt LE, Rasmussen R, Mackinnon NC, Vossen JF, Pelham TW. Effects of hot or cold water immersion and modified proprioceptive neuromuscular facilitation flexibility exercise on hamstring length. J Athl Train. 2001;36:(1):16-9.
15. Magalhães FEX, Mesquita Júnior AR, Meneses HTS, Santos RPM, Rodrigues EC, Gouveia SSV, Gouveia GPM, Orsini M, Bastos $\mathrm{VHV}$, Machado DCD. Comparison of the effects of hamstring stretching using proprioceptive neuromuscular facilitation with prior application of cryotherapy or ultrasound therapy. J Phys Ther Sci. 2015:27:1549-53. https://doi.org/10.1589/jpts.27.1549

16. Hurley MV, Scott DL, Rees J, Newham DJ. Sensorimotor changes and functional performance in patients with knee osteoarthritis. Ann Rheum Dis. 1997;56:641-8. https://doi.org/10.1136/ard.56.11.641

17. Signori LU, Voloski FRS, Kerkhoff AC, Brignoni L, Plentz RDM. Efeito de agentes térmicos aplicados previamente a um programa de alongamentos na flexibilidade dos músculos isquiotibiais encurtados. Rev Bras Med Esporte. 2008;14(4):328-31. https://doi. org/10.1590/S1517-86922008000400001

18. Knight KL. Crioterapia no tratamento das lesões desportivas. São Paulo: Manole; 2000.

19. Peres SE, Draper DO, Knight KL, Ricard MD. Pulsed Shortwave Diathermy and Prolonged Long-Duration Stretching Increase Dorsiflexion Range of Motion More Than Identical Stretching Without Diathermy. J Athl Train. 2002;37(1):43-50.

20. Silva KNG, Pinfildi CE, Machado FA, Lotufo RF, Cohen M, Peccin MS. Influência do frio no pico de torque da musculatura flexora do joelho. In: Anais do XII Congresso Brasileiro de Biomecânica. Rio Claro: Unesp; 2007.

21. Crystal NJ, Townson DH, Cook SB, LaRoche DP. Effect of cryotherapy on muscle recovery and inflammation following a bout of damaging exercise. Eur J Appl Physiol. 2013;113:2577-86. https://doi. org/10.1007/s00421-013-2693-9

22. Mustalampi S, Ylinen J, Kautiainen H, Weir A, Häkkinen A. Acute effects of cold pack on mechanical properties of the quadriceps muscle in healthy subjects. Phys Ther Sport. 2012;13(4):265-9. https://doi.org/10.1016/j.ptsp.2012.02.001

23. Akyol Y, Durmus D, Alayli G, Tander B, Bek Y, Canturk F, Tastan Sakarya S. Does short-wave diathermy increase the effectiveness of isokinetic exercise on pain, function, knee muscle strength, quality of life, and depression in the patients with knee osteoarthritis? Eur J Phys Rehabil Med. 2010;46:325-36.

24. Cetin N, Aytar A, Atalay A, Akman MN. Comparing hot pack, shortwave diathermy, ultrasound, and TENS on isokinetic strength, pain and functional status of women with osteoarthritic knees: a singleblind, randomized, controlled trial. Am J Phys Med Rehabil. 2008;87: 443-51. https://doi.org/10.1097/PHM.0b013e318174e467

25. Rattanachaiyanont M, Kuptniratsaikul V. No additional benefit of shortwave diathermy over exercise program for knee osteoaerthritis in peri/post menopausal women: an equivalence trial. Osteoarthritis Cartilage. 2008;16(7):823-8. https://doi.org/10.1016/j.joca.2007. 10.013

26. Petrofsky JS, Laymon M, Lee H. Effect of heat and cold on tendon flexibility and force to flex the human knee. Med Sci Monit. 2013; 19: 661-7. https://doi.org/10.12659/MSM.889145

27. Carvalho ACG, Paula KC, Azevedo TMC, Nóbrega ACLN. Relação entre flexibilidade e força muscular em adultos jovens de ambos os sexos. Rev Bras Med Esporte. 1998;4(1):2-8. https://doi.org/ 10.1590/S1517-86921998000100002 